\title{
Constraining Relativistic Generalizations of Modified Newtonian Dynamics with Gravitational Waves
}

\author{
Paul M. Chesler情 and Abraham Loeb \\ Black Hole Initiative, Harvard University, Cambridge, MA 02138, USA
}

(Dated: July 3, 2017)

\begin{abstract}
In the weak-field limit of General Relativity, gravitational waves obey linear equations and propagate at the speed of light. These properties of General Relativity are supported by the observation of ultra high energy cosmic rays as well as by LIGO's recent detection of gravitation waves. We argue that two existing relativistic generalizations of Modified Newtonian Dynamics, namely Generalized Einstein-Aether theory and BIMOND, display fatal inconsistencies with these observations.
\end{abstract}

Introduction.-With LIGO's discovery of black hole mergers [1, 2], gravitational waves have advanced from a theoretical prediction of General Relativity (GR) to an experimental detection. Gravitational waves also exist in modified theories of gravity and can have distinguishing features which can be used to constrain the theoretical space of allowable theories. Of particular interest are relativistic generalizations of Modified Newtonian Dynamics (MOND) 3] (henceforth referred to as MONDian theories). These are modified theories of gravity designed to emulate the effects of dark matter without actually having dark matter (see 4 for a review).

At the core of MONDian theories is the assumption that in the limit of sufficiently weak acceleration, gravitational dynamics becomes non-linear, with the nonlinearities tailored to yield flat rotation curves of galaxies [25]. In the weak-field quasi-static limit, where the metric can be written $d s^{2}=-(1+2 \Phi) d t^{2}+(1+2 \Psi) d \boldsymbol{x}^{2}$, the field equations must reduce to $\Psi=-\Phi$ and [4]

$$
\nabla \cdot\left[\mu\left(\frac{|\nabla \Phi|}{a_{0}}\right) \nabla \Phi\right]=4 \pi G \rho,
$$

where $G$ is Newton's constant, $a_{0} \approx 10^{-10} \mathrm{~m} / \mathrm{s}^{2}$, and $\rho$ is the matter density. The MOND function $\mu(x)$ satisfies $\mu(x) \rightarrow 1$ when $x \gg 1$, so that Newton's law of gravity is recovered in the strong acceleration limit, and $\mu(x) \rightarrow x$ when $x \ll 1$, which yields flat rotation curves of galaxies at large distances from matter sources. Hence, the equations of motion for the potential become non-linear when $|\nabla \Phi| \ll a_{0}$. This stands in stark contrast to GR, where the weak-field limit is governed by linear equations of motion. MONDian theories of gravity include TeVeS [5], generalized Einstein-Aether theories [6, bimetric theories (BIMOND) [7, 8, and non-local theories [9, 10. Recently Verlinde [11] suggested that similar modifications can naturally occur in entropic gravity 12 .

MONDian modifications to GR can potentially alter gravitational wave physics in at least two ways. First, since MOND is an acceleration based modification of gravity, MONDian theories can violate the equivalence principle. A consequence of this is that gravitational waves can propagate subluminally. Second, since MONDian theories are non-linear in the weak field limit, gravitational waves can be governed by non-linear equations, even in the weak-field limit. As we elaborate on below, these features have unsavory consequences and can be used to restrict the set of allowed MONDian theories.

If gravitational waves propagate non-luminally, the arrival time for electromagnetic and gravitational signals from astrophysical events can be different [13, 14]. Moreover, as was pointed out long ago [15], if the speed of gravitational waves is $c_{\mathrm{g}}<1$ (in units where $c=1$ ), then high energy cosmic rays traveling at speed $v \rightarrow 1$ will lose energy via the emission of gravitational Cherenkov radiation, with an energy loss rate dependent on the difference $1-c_{\mathrm{g}}$. The observation of high energy cosmic rays on earth, combined with an estimate of their distance of propagation, then sets lower bounds on $c_{\mathrm{g}}$, which have been estimated to be $1-c_{\mathrm{g}} \lesssim 10^{-15}[15$, 16]. In the MOND limit of the Einstein-Aether theory of Ref. [6], we demonstrate that the speed of gravitational waves depends on the local gravitational potential and generically cannot be set equal to the speed of light, and that Cherenkov losses are unavoidable without making the theory pathological. These features make this theory an unacceptable theory of gravity.

Second, if gravitational wave dynamics are non-linear in the weak-field limit, gravitational waves emitted in black hole merger events can interact with themselves as well as with other gravitational waves, effectively scrambling the structure of the original waveforms as they propagate to earth. LIGO's recent observation of GW150914 had a gravitational waveform completely consistent with GR [1], suggesting no such scrambling effect. A natural expectation is therefore that gravitational waves must satisfy linear equations of motion in the weak-field limit of any acceptable theory of gravity.

We argue that interactions between gravitational wave packets in the weak-field limit of BIMOND 8 alters the structure of the original waveforms and can even lead to singular evolution. Therefore, if BIMOND reduces to GR in the strong field limit - and thereby yields the same initial gravitational waveforms as GR in merger events - the waveforms observed far away would not look anything like those predicted by GR. In BIMOND we argue non-linear interactions become important at distances on the order $0.3 \mathrm{Gpc}$ from merger events. In contrast, grav- 
itational waves from GW150914 are estimated to have propagated 0.4 Gpc. Our results and the experimental data from LIGO suggest that BIMOND in its present form is not an acceptable theory of modified gravity.

Generalized Einstein-Aether Theories.-In addition to the metric $g_{\mu \nu}$, Einstein-Aether theories contain a time-like vector field $A^{\mu}$ which satisfies $A^{2}=-1$ and defines a preferred frame. Following Ref. 6] we consider the gravitational action,

$S=\frac{1}{16 \pi G} \int d^{4} x \sqrt{g}\left[R+M^{2} \mathcal{F}\left(\frac{\mathcal{K}}{M^{2}}\right)+\lambda\left(A^{2}+1\right)\right]+S_{\text {mat }}$,

where $\mathcal{K} \equiv \mathcal{K}^{\alpha \beta}{ }_{\gamma \sigma} \nabla_{\alpha} A^{\gamma} \nabla_{\beta} A^{\sigma}$ is a quadratic function of derivatives of $A^{\mu}, \lambda$ is a Lagrange multiplier which enforces the constraint $A^{2}=-1, M$ is a constant with dimensions of mass, and $S_{\text {mat }}$ is the matter action. The function $\mathcal{F}$ determines the function $\mu$ in $(1)$. The most general expression for $\mathcal{K}_{\mu \nu}^{\alpha \beta}$ involving no derivatives reads

$$
\mathcal{K}_{\gamma \sigma}^{\alpha \beta} \equiv c_{1} g^{\alpha \beta} g_{\gamma \sigma}+c_{2} \delta_{\gamma}^{\alpha} \delta_{\sigma}^{\beta}+c_{3} \delta_{\sigma}^{\alpha} \delta_{\gamma}^{\beta}+c_{4} A^{\alpha} A^{\beta} g_{\sigma \gamma},
$$

where the $c_{i}$ are dimensionless constants. Following 6] we shall set $M=\epsilon^{2} a_{0}$ with $\epsilon$ a bookkeeping parameter which can be set to one after all calculations.

The Einstein-Aether equations of motion read

$$
\begin{aligned}
R_{\mu \nu}-\frac{1}{2} R g_{\mu \nu} & =\mathcal{T}_{\mu \nu}+8 \pi G T_{\mu \nu}^{\text {mat }} \\
\nabla_{\alpha}\left[\mathcal{F}^{\prime} J_{\beta}^{\alpha}\right]-\mathcal{F}^{\prime} y_{\beta} & =2 \lambda A_{\beta}
\end{aligned}
$$

with $T_{\mu \nu}^{\text {mat }}$ the matter stress and $\mathcal{T}_{\alpha \beta}$ the vector stress,

$$
\begin{aligned}
\mathcal{T}_{\alpha \beta} & =\frac{1}{2} \nabla_{\sigma}\left\{\mathcal{F}^{\prime}\left[J_{(\alpha}{ }^{\sigma} A_{\beta)}-J_{(\alpha}^{\sigma} A_{\beta)}-J_{(\alpha \beta)} A^{\sigma}\right]\right\} \\
& -\mathcal{F}^{\prime} Y_{\alpha \beta}+\frac{1}{2} g_{\alpha \beta} M^{2} \mathcal{F}+\lambda A_{\alpha} A_{\beta}
\end{aligned}
$$

with

$$
\begin{aligned}
Y_{\alpha \beta}= & -c_{1}\left[\nabla_{\nu} A_{\alpha} \nabla^{\nu} A_{\beta}-\nabla_{\alpha} A_{\nu} \nabla_{\beta} A^{\nu}\right] \\
& -c_{4}\left(A \cdot \nabla A_{\alpha}\right)\left(A \cdot \nabla A_{\beta}\right), \\
J_{\sigma}^{\alpha}= & 2 \mathcal{K}_{\sigma \gamma}^{\alpha \beta} \nabla_{\beta} A^{\gamma}, \\
y_{\beta}= & 2 c_{4} \nabla_{\beta} A_{\mu}\left(A \cdot \nabla A^{\mu}\right) .
\end{aligned}
$$

We wish to study Einstein-Aether waves in the MOND limit, particularly waves propagating in the background of a weak, static and slowly varying gravitational field.
Our goal here is to demonstrate that the propagation speeds depend on the local background fields and cannot be set equal to the speed of light for all modes. To this end let us first consider static, weak field, and slowly varying solutions to the Einstein-Aether system. Following [6] we consider the ansatz,

$$
\begin{aligned}
g_{\mu \nu}(t, \boldsymbol{x}) & =\eta_{\mu \nu}-2 \epsilon \Phi(\epsilon \boldsymbol{x}) \delta_{\mu \nu}, \\
A_{\mu}(t, \boldsymbol{x}) & =[-1+\epsilon \Phi(\epsilon \boldsymbol{x})] \delta_{\mu 0},
\end{aligned}
$$

and solve the equations of motion in the $\epsilon \rightarrow 0$ limit. The above ansatz satisfies $A^{2}=-1+O\left(\epsilon^{2}\right)$. In the $\epsilon \rightarrow 0$ limit the Einstein-Aether equations of motion (4) reduce to the MOND equation (1) with $\mu(x)=x$ provided [6]

$$
\mathcal{F}(x)=\frac{1}{-c_{1}+c_{4}}\left[2 x-\frac{4}{3 \sqrt{-c_{1}+c_{4}}} x^{3 / 2}\right] \text {. }
$$

We note that the Einstein-Aether theory can be made GR-compatible in the strong field limit if one sets $\mathcal{F}(x)=$ $\xi x$. By setting $\xi \rightarrow 0$, the vector stress (5) can be made arbitrarily small. Hence, constraints from strong field gravity, such as those studied in [17 19], can be satisfied.

Consider now the ansatz

$$
\begin{aligned}
g_{\mu \nu}(t, \boldsymbol{x}) & =\eta_{\mu \nu}-2 \epsilon \Phi(\epsilon \boldsymbol{x}) \delta_{\mu \nu}+\zeta h_{\mu \nu} e^{-i \omega t+i \boldsymbol{k} \cdot \boldsymbol{x}}, \\
A_{\mu}(t, \boldsymbol{x}) & =[-1+\epsilon \Phi(\epsilon \boldsymbol{x})] \delta_{\mu 0}+\zeta a_{\mu} e^{-i \omega t+i \boldsymbol{k} \cdot \boldsymbol{x}}
\end{aligned}
$$

which describes small perturbations propagating on top of the static background potential $\Phi$. Here $\zeta$ is another bookkeeping parameter which parameterizes the strength of the propagating modes. We shall consider the $\epsilon \rightarrow 0$ limit with $\zeta \ll \epsilon^{2}$. In this limit the exponentials vary in space much more rapidly than the potential. Note that $h_{\mu \nu}$ and $a_{\mu}$ also depend on $\boldsymbol{x}$. However, this dependence can be neglected at leading order. For simplicity we assume the potential vanishes as the point $\boldsymbol{x}$ of interest and that $\boldsymbol{k}$ and $\nabla \Phi$ point in the same direction at $\boldsymbol{x}$.

The equations of motion in the MOND limit for $h_{\mu \nu}$ and $a_{\mu}$, as well as the dispersion relation $\omega(k)$, follow from substituting the ansatz (9) into (4) with $\mathcal{F}$ given by Eq. (8). With $\zeta \ll \epsilon^{2}$ and the presence of the background potential, the equations of motion for $h_{\mu \nu}$ and $a_{\mu}$ are linear. There are a total of five propagating modes, including two tensor modes, two vector modes, and one scalar mode. We find linear dispersion relations $\omega=c_{\mathrm{g}} k$ for all modes, with propagation speeds,

$$
\begin{aligned}
c_{\text {tensor }}^{2} & =\frac{-c_{1}+c_{4}}{\left(c_{1}+2 c_{3}+c_{4}\right)-2\left(c_{1}+c_{3}\right)|\nabla \Phi| / a_{0}}, \\
c_{\text {vector }}^{2} & =\frac{c_{3}^{2}-c_{1} c_{4}+\left(c_{1}^{2}-c_{3}^{2}\right)|\nabla \Phi| / a_{0}}{\left(-c_{1}+c_{4}\right)\left[c_{1}+2 c_{3}+c_{4}-2 c_{1}\left(c_{1}+c_{3}\right)|\nabla \Phi| / a_{0}\right]}, \\
c_{\text {scalar }}^{2} & =\frac{2\left(-c_{1}+c_{4}\right)\left(c_{1}+c_{2}+c_{3}\right)\left(1-|\nabla \Phi| / a_{0}\right)|\nabla \Phi| / a_{0}}{\left(1-2|\nabla \Phi| / a_{0}\right)\left[c_{1}+2 c_{3}+c_{4}-2\left(c_{1}+c_{3}\right)|\nabla \Phi| / a_{0}\right]\left[2 c_{1}+3 c_{2}+c_{3}-c_{4}-\left(c_{1}+3 c_{2}+c_{3}\right)|\nabla \Phi| / a_{0}\right]}
\end{aligned}
$$


Note that the propagation speeds depend on the local value of the potential gradient, $|\nabla \Phi| / a_{0}$.

Setting the speed of all excitations equal to the speed of light will guarantee no cosmic ray energy loss via gravitational Cherenkov radiation. Let us focus on the tensor and scalar modes. Inspection of Eq. 10 shows that setting $c_{\text {tensor }}=1$ requires $c_{3}=-c_{1}$. In contrast, no choice of $c_{i}$ can set $c_{\text {scalar }}=1$. Nevertheless, the scalar mode can be made non-propagating, meaning $c_{\text {scalar }}=0$, if in addition to $c_{3}=-c_{1}$ we set $c_{2}=0$.

There is another reason that one should set $c_{3}=-c_{1}$. As we shall discuss further below in the next section, in the absence of background potentials (i.e. $\Phi=0$ ) it is desirable that gravitational waves should satisfy linear equations in the weak field limit. For the tensor modes this can only be accomplished if $c_{3}=-c_{1}[26$.

It is easy to see that the Einstein-Aether theory with $c_{3}=-c_{1}$ and $c_{2}=0$ develops pathologies, even with regular initial data. The pathologies are essentially the MONDian equivalent of those found in the EinsteinAether theory studied in [20. In particular, with regular initial data the vector field will generically evolve to a singular solution. To see this, first note that with $c_{3}=-c_{1}$ and $c_{2}=0$ we have,

$$
\mathcal{K}=-\frac{c_{1}}{2} F_{\mu \nu} F^{\mu \nu}+c_{4}(A \cdot \nabla A)^{2},
$$

where $F_{\mu \nu}=\partial_{\mu} A_{\nu}-\partial_{\nu} A_{\mu}$ is the field strength of $A$. Now consider pure gauge solutions in which $F_{\mu \nu}=0$. From $F_{\mu \nu}=0$, it follows that $\nabla_{\alpha} A^{2}=2 A \cdot \nabla A_{\alpha}=0$, meaning that $A$ is tangent to a congruence of time-like geodesics. Such vector fields have $\mathcal{K}=0, J_{\mu \nu}=Y_{\mu \nu}=0$, and are exact solutions to the equation of motion (4). They also have vanishing stress $\mathcal{T}^{\mu \nu}$, meaning they do not back react on the geometry. Consider then a solution to the Einstein equations sourced by some matter distribution. Generically the congruence of geodesics will form caustics. When this happens $\nabla_{\alpha} A_{\beta}$ will become singular, and the classical theory will break down. Therefore, demanding no Cherenkov energy losses - by setting $c_{3}=-c_{1}$ and $c_{2}=0$ - results in a non-viable theory.

BIMOND. - In addition to the physical metric $g_{\mu \nu}$, BIMOND [7, 8, contains an additional metric $\hat{g}_{\mu \nu}$. The difference between the Christoffel symbols associated with the two metrics,

$$
C_{\mu \nu}^{\lambda}=\Gamma_{\mu \nu}^{\lambda}-\hat{\Gamma}_{\mu \nu}^{\lambda},
$$

is itself a tensor. We will refer to $C_{\mu \nu}^{\lambda}$ as the acceleration tensor. The MOND limit is defined by $\left|C_{\mu \nu}^{\lambda}\right| \lesssim a_{0}$. In the limit $\left|C_{\mu \nu}^{\lambda}\right| \gg a_{0}$, interactions between the two metrics are assumed to vanish and the dynamics of $g_{\mu \nu}$ are governed by GR.

Before writing down the action of BIMOND, let us first estimate the magnitude of the acceleration tensor associated with gravitational waves emitted by merging black holes at a distance $r$ away from an observer. In the weak-field limit, the acceleration tensor scales as $h / \lambda$ where $h$ is the amplitude of the wave and $\lambda$ its wavelength. For equal mass binary black hole mergers the amplitude roughly scales like $h \sim G M / r$, with $M$ being the mass of the binary. Likewise, for equal mass mergers the wavelength of emitted radiation should be set by the Schwarzschild radius, implying that $\lambda \sim G M$. We therefore obtain,

$$
\left|C_{\mu \nu}^{\lambda}\right| \sim \frac{1}{r} .
$$

This is equal to $a_{0}$ when $r \sim 0.3 \mathrm{Gpc}$. This suggests that gravitational waves from GW150914, which are believed to have originated $0.4 \mathrm{Gpc}$ away, have probed the MONDian limit. Indeed, for GW150914 the observed amplitude of the gravitational waves was $h \sim 10^{-21}$ while the wavelength was $\lambda \sim 5 \times 10^{6} \mathrm{~m}$, which yields $h / \lambda \approx 0.2 a_{0}$. Moreover, it is likely that these gravitational waves propagated through cosmic voids, where the acceleration tensor due to quasi-static matter sources is less than the acceleration produced by the gravitational waves. We therefore chose to study gravitational waves propagating in the absence of background potentials. The equations of motion for such gravitational waves in BIMOND were derived in Ref. 8, as we briefly sketch below.

Following Milgrom [8, we consider the gravitational action

$$
S_{\text {grav }}=\frac{1}{16 \pi G} \int d^{4} x\left[\alpha \sqrt{g} R+\beta \sqrt{\hat{g}} \hat{R}+M^{2} \mathcal{F}\right],
$$

where again $M=\epsilon a_{0}$ with $\epsilon$ a bookkeeping parameter. Here $R$ and $\hat{R}$ are the Ricci scalars of $g_{\mu \nu}$ and $\hat{g}_{\mu \nu}$, respectively, and $\alpha$ and $\beta$ are constants. We shall consider the case $\alpha \neq-\beta$. $\mathcal{F}$ characterizes the interactions between the two metrics. Derivative interactions between $g_{\mu \nu}$ and $\hat{g}_{\mu \nu}$ are assumed to solely be contained in the dimensionless scalar arguments $\frac{1}{M^{2}} g^{\mu \nu} \Upsilon_{\mu \nu}$ and $\frac{1}{M^{2}} \hat{g}^{\mu \nu} \Upsilon_{\mu \nu}$ with

$$
\Upsilon_{\mu \nu} \equiv C_{\mu \lambda}^{\gamma} C_{\nu \gamma}^{\lambda}-C_{\mu \nu}^{\gamma} C_{\lambda \gamma}^{\lambda} .
$$

Note that additionally $\mathcal{F}$ can depend on the nonderivative interactions such as $g \hat{g}$ or $g^{\mu \nu} \hat{g}_{\mu \nu}$.

In the weak field limit we can write

$$
g_{\mu \nu}=\eta_{\mu \nu}+\epsilon h_{\mu \nu}, \quad \hat{g}_{\mu \nu}=\eta_{\mu \nu}+\epsilon \hat{h}_{\mu \nu} .
$$

We wish to obtain the equations of motion for $h_{\mu \nu}$ and $\hat{h}_{\mu \nu}$ at first order in $\epsilon$. At first order any dependence on non-derivative interactions between $h_{\mu \nu}$ and $\hat{h}_{\mu \nu}$ can be neglected. Likewise, in this limit $\frac{1}{M^{2}} g^{\mu \nu} \Upsilon_{\mu \nu}=$ $\frac{1}{M^{2}} \hat{g}^{\mu \nu} \Upsilon_{\mu \nu} \equiv \frac{1}{M^{2}} \Upsilon$ where,

$$
\Upsilon \equiv \eta^{\mu \nu} \Upsilon_{\mu \nu}
$$


Therefore, in the weak field limit we can take,

$$
\mathcal{F}=\mathcal{F}\left(-\Upsilon / 2 M^{2}\right) .
$$

For quasi-static geometries (and $\alpha \neq-\beta$ ) MOND phenomenology is obtained when 8 ]

$$
\mathcal{F}^{\prime}(x)+\frac{\alpha \beta}{\alpha+\beta} \sim \sqrt{x}, \quad x \ll 1 .
$$

For GW150914 we estimate $\Upsilon \sim 0.04 a_{0}^{2}$. Hence we employ 19. for studying gravitational waves.

The equations of motion for the metric perturbations can easily be derived from the action (14) and Eqs. (18) and (19). Define,

$$
\Delta h_{\mu \nu} \equiv h_{\mu \nu}-\hat{h}_{\mu \nu}, \quad s_{\mu \nu} \equiv \alpha h_{\mu \nu}+\beta \hat{h}_{\mu \nu} .
$$

Since $\mathcal{F}$ only depends on $\Delta h_{\mu \nu}$, the weak field action for $s_{\mu \nu}$ is simply that of linearized Einstein equations, meaning $s_{\mu \nu}$ satisfies the linearized Einstein equations. In contrast, up to inconsequential surface terms the weak field action for $\Delta h_{\mu \nu}$ is [8],

$$
S \propto \int d^{4} x(-\Upsilon)^{3 / 2} .
$$

It follows that $\Delta h_{\mu \nu}$ is governed by non-linear equations.

To illustrate the non-linear nature of the equations of motion and the resulting instabilities they possess, let us focus on waves propagating in the $z$ direction with a single excited mode $\Delta h_{x y}(t, z)$, with $x, y$ the two transverse directions to $z$. In this case, the action (21) leads to the equation of motion,

$$
\partial_{\mu}\left\{\left|\partial \Delta h_{x y}\right| \partial^{\mu} \Delta h_{x y}\right\}=0,
$$

where $\left|\partial \Delta h_{x y}\right| \equiv \sqrt{-\left(\partial_{t} \Delta h_{x y}\right)^{2}+\left(\partial_{z} \Delta h_{x y}\right)^{2}}$.

As was pointed out by Milgrom 8, any function of $t \pm z$ is an exact solution to Eq. (22). Nevertheless Eq. (22) is problematic for gravitational wave physics for several reasons. First and foremost, Eq. 22 is non-linear and consequently superpositions of solutions propagating in opposite directions are not themselves solutions. This means that gravitational waves propagating in different directions will interact with each other, with the outgoing waveforms being modified by the interaction. This alone would suggest that gravitational waves in BIMOND originating from merger events should not resemble those in GR, which is in contradiction with data from LIGO.

Moreover, the violations of the superposition principle can be violent, with interactions resulting in singularities. In particular, at points in which $2\left(\partial_{t} \Delta h_{x y}\right)^{2}=$ $\left(\partial_{z} \Delta h_{x y}\right)^{2}$, Eq. 22 implies that $\partial_{t}^{2} \Delta h_{x y}$ generically diverges. For example, a superposition of two plane waves initially propagating in the $\pm z$ directions can have divergent $\partial_{t}^{2} \Delta h_{x y}$. We have also numerically solved Eq. 222 for the collision of two initially well separated Gaussian wave packets propagating towards each other at the speed of light. We have found that as soon as the tails of the Gaussian begin to overlap, interactions naturally lead to points in which $\left(\partial_{t} \Delta h_{x y}\right)^{2}=2\left(\partial_{z} \Delta h_{x y}\right)^{2}$ and correspondingly, $\Delta h_{x y}$ becoming singular [27]. While its possible that higher order corrections to the scaling relation (19) may ameliorate the formation of singularities, the structure of gravitational wave forms will still be dramatically altered by interactions with background gravitational waves, and hence will not resemble wave forms in GR. These features make BIMOND in its present form an unsatisfactory theory of modified gravity.

Discussion.-In this paper we have argued that gravitational waves should obey two simple principles: (i) they should propagate at the speed of light and (ii) they should obey linear equations in the weak-field limit. The first condition follows from considerations of energy loss of cosmic rays via gravitational Cherenkov radiation [15], while the second is supported by recent observations of gravitational waves by LIGO. It is striking how these two simple principles constrain the space of allowed MONDian theories of gravity. It is worth noting that gravitational waves in TeVeS also generically propagate at speeds different from the speed of light [21].

We note that Milgrom [22] has argued that MONDian effects can drastically reduce gravitational Cherenkov energy losses. Energy loss from gravitational Cherenkov radiation is dominated by the emission of radiation with momentum $k \sim p$, with $p$ the momentum of the cosmic ray [15. Milgrom suggested that MONDian effects can result in a large effective size of cosmic rays and that radiation emitted from different points will deconstructively interfere, resulting in an effective momentum cutoff $k_{\max } \ll p$ and hence much weaker energy loss from gravitational Cherenkov radiation. For steady-state sources of radiation this is clearly the case, since the transverse size $R$ of a steady-state classical source cuts off the spectrum of excited modes at $k \propto 1 / R$. However, this argument cannot be correct for non-steady-state sources of radiation. First, the emission of Cherenkov radiation is similar to an unstable particle decaying. Indeed, both processes are described by the same Feynman diagrams. Milgrom's argument would suggest that large composite particles should be stable to decay via the emission of short wavelength radiation. The flaw in this argument lies in the fact that it neglects the (large) recoil experienced by cosmic rays when radiation is emitted. Simply put, this argument cannot apply to gravitational Cherenkov radiation irrespective of any effective size induced by MONDian physics.

Acknowledgments.-This work was supported in part by the Black Hole Initiative at Harvard University, which is funded by a grant from the John Templeton Foundation. We thank Guy Moore for helpful conversa- 
tions.

* Electronic address: pchesler@g.harvard.edu

† Electronic address: aloeb@cfa.harvard.edu

[1] Virgo, LIGO Scientific Collaboration, B. P. Abbott et al., "Observation of Gravitational Waves from a Binary Black Hole Merger," Phys. Rev. Lett. 116 (2016) no. 6, 061102, arXiv:1602.03837 [gr-qc]

[2] Virgo, LIGO Scientific Collaboration, B. P. Abbott et al., "GW151226: Observation of Gravitational Waves from a 22-Solar-Mass Binary Black Hole Coalescence," Phys. Rev. Lett. 116 (2016) no. 24, 241103. arXiv:1606.04855 [gr-qc]

[3] M. Milgrom, "A Modification of the Newtonian dynamics as a possible alternative to the hidden mass hypothesis," Astrophys. J. 270 (1983) 365-370

[4] B. Famaey and S. McGaugh, "Modified Newtonian Dynamics (MOND): Observational Phenomenology and Relativistic Extensions," Living Rev. Rel. 15 (2012) 10 , arXiv:1112.3960 [astro-ph.CO].

[5] J. D. Bekenstein, "Relativistic gravitation theory for the MOND paradigm," Phys. Rev. D70 (2004) 083509 arXiv:astro-ph/0403694 [astro-ph] [Erratum: Phys. Rev.D71,069901(2005)].

[6] T. G. Zlosnik, P. G. Ferreira, and G. D. Starkman, "Modifying gravity with the Aether: An alternative to Dark Matter," Phys. Rev. D75 (2007) 044017, arXiv:astro-ph/0607411 [astro-ph]

[7] M. Milgrom, "Bimetric MOND gravity," Phys. Rev. D80 (2009) 123536, arXiv:0912.0790 [gr-qc].

[8] M. Milgrom, "Gravitational waves in bimetric MOND," Phys. Rev. D89 (2014) no. 2, 024027, arXiv:1308.5388 [gr-qc]

[9] C. Deffayet, G. Esposito-Farese, and R. P. Woodard, "Nonlocal metric formulations of MOND with sufficient lensing," Phys. Rev. D84 (2011) 124054 arXiv:1106.4984 [gr-qc]

[10] C. Deffayet, G. Esposito-Farese, and R. P. Woodard, "Field equations and cosmology for a class of nonlocal metric models of MOND," Phys. Rev. D90 (2014) no. 6, 064038, arXiv:1405.0393 [astro-ph.C0] [Addendum: Phys. Rev.D90,no.8,089901(2014)].

[11] E. P. Verlinde, "Emergent Gravity and the Dark Universe," arXiv:1611.02269 [hep-th]

[12] E. P. Verlinde, "On the Origin of Gravity and the Laws of Newton," JHEP 04 (2011) 029, arXiv : 1001.0785 [hep-th]

[13] E. O. Kahya and R. P. Woodard, "A Generic Test of Modified Gravity Models which Emulate Dark Matter,"
Phys. Lett. B652 (2007) 213-216, arXiv:0705.0153 [astro-ph]

[14] E. O. Kahya, "A Useful guide for gravitational wave observers to test modified gravity models," Phys. Lett. B701 (2011) 291-295, arXiv: 1001.0725 [gr-qc]

[15] G. D. Moore and A. E. Nelson, "Lower bound on the propagation speed of gravity from gravitational Cherenkov radiation," JHEP 09 (2001) 023 arXiv:hep-ph/0106220 [hep-ph]

[16] J. W. Elliott, G. D. Moore, and H. Stoica, "Constraining the new Aether: Gravitational Cerenkov radiation," JHEP 08 (2005) 066, arXiv:hep-ph/0505211 [hep-ph]

[17] M. Bonetti and E. Barausse, "Post-Newtonian constraints on Lorentz-violating gravity theories with a MOND phenomenology," Phys. Rev. D91 (2015) 084053 arXiv:1502.05554 [gr-qc]. [Erratum: Phys. Rev.D93,029901(2016)].

[18] K. Yagi, D. Blas, N. Yunes, and E. Barausse, "Strong Binary Pulsar Constraints on Lorentz Violation in Gravity," Phys. Rev. Lett. 112 (2014) no. 16, 161101 arXiv:1307.6219 [gr-qc].

[19] N. Yunes, K. Yagi, and F. Pretorius, "Theoretical Physics Implications of the Binary Black-Hole Mergers GW150914 and GW151226," Phys. Rev. D94 (2016) no. 8, 084002, arXiv:1603.08955 [gr-qc]

[20] T. Jacobson and D. Mattingly, "Gravity with a dynamical preferred frame," Phys. Rev. D64 (2001) 024028, arXiv:gr-qc/0007031 [gr-qc].

[21] E. Sagi, "Propagation of gravitational waves in generalized TeVeS," Phys. Rev. D81 (2010) 064031 arXiv:1001.1555 [gr-qc].

[22] M. Milgrom, "Gravitational Cherenkov losses in MOND theories," Phys. Rev. Lett. 106 (2011) 111101, arXiv:1102.1818 [astro-ph.CO]

[23] M. Milgrom, "Dynamics with a non-standard inertia-acceleration relation: an alternative to dark matter," Annals Phys. 229 (1994) 384-415. arXiv:astro-ph/9303012 [astro-ph]

[24] M. Milgrom, "MOND-particularly as modified inertia," Acta Phys. Polon. B42 (2011) 2175-2184, arXiv:1111.1611 [astro-ph.CO]

[25] We note that there also exist formulations of MOND which modify interia instead of gravity [23, 24]. In this paper we only consider modifications of gravity.

[26] We note that even after setting $c_{3}=-c_{1}$, the scalar and vector modes still satisfy non-linear equations.

[27] In the absence of background potentials, similar instabilities also exist in the vector and scalar modes in above Einstein-Aether theory. 